\title{
Non-Hamiltonian Actions
}

\section{and Lie-Algebra Cohomology of Vector Fields ${ }^{\star}$}

\author{
Roberto FERREIRO PÉREZ ${ }^{\dagger}$ and Jaime MUÑOZ MASQUÉ $\ddagger$ \\ † Departamento de Economía Financiera y Contabilidad I, Facultad de Ciencias Económicas \\ y Empresariales, UCM, Campus de Somosaguas, 28223-Pozuelo de Alarcón, Spain \\ E-mail: roferreiro@ccee.ucm.es \\ ¥ Instituto de Física Aplicada, CSIC, C/ Serrano 144, 28006-Madrid, Spain \\ E-mail: jaime@iec.csic.es
}

Received April 03, 2009, in final form June 08, 2009; Published online June 16, 2009

doi:10.3842/SIGMA.2009.063

\begin{abstract}
Two examples of Diff ${ }^{+} S^{1}$-invariant closed two-forms obtained from forms on jet bundles, which does not admit equivariant moment maps are presented. The corresponding cohomological obstruction is computed and shown to coincide with a nontrivial Lie algebra cohomology class on $H^{2}\left(\mathfrak{X}\left(S^{1}\right)\right)$.
\end{abstract}

Key words: Gel'fand-Fuks cohomology; moment mapping; jet bundle

2000 Mathematics Subject Classification: 58D15; 17B56; 22E65; 53D20; 53D30; 58A20

\section{Introduction}

Let $p: E \rightarrow M$ be a bundle over a compact, oriented $n$-manifold $M$ without boundary. In [1] the forms on the jet bundle of degree greater than the dimension of the base manifold are interpreted as differential forms on the space of sections by means of the integration map $\Im: \Omega^{n+k}\left(J^{r} E\right) \rightarrow$ $\Omega^{k}(\Gamma(E))$ (see Section 2 for the details).

In particular, if $\alpha$ is a closed $(n+2)$-form on $J^{r} E$ and is invariant under the action of a group $\mathcal{G}$ on $E$, then $\Im[\alpha]$ determines a $\mathcal{G}$-invariant closed two-form on $\Gamma(E)$. In [1] this is applied to the case of connections on a principal bundle, and in [3] to the case of Riemannian metrics. Moreover, in those cases canonical moment maps for these forms are obtained. The moment maps are obtained using the fact that the closed two-forms came from characteristic classes of an invariant connection, and the moment maps came from the equivariant characteristic classes.

In general, however, if $\alpha \in \Omega^{n+2}\left(J^{r} E\right)$ is closed and $\mathcal{G}$-invariant, then $\Im[\alpha]$ does not admit a moment map necessarily because cohomological obstructions could exist (see Section 3 for the details). In this paper we present two examples where this happens. In the first example we consider maps $S^{1} \rightarrow S^{1}$ and the action of the orientation preserving diffeomorphisms on $S^{1}$. We define an invariant closed two-form on the space $\mathfrak{A}=\left\{u: S^{1} \rightarrow S^{1}: \dot{u}(t) \neq 0, \forall t \in S^{1}\right\}$, and we show that the obstruction to the existence of an equivariant moment map is a nontrivial class in the Lie algebra cohomology of $\mathfrak{X}\left(S^{1}\right)$.

In the second example we consider regular closed plane curves and the same group as in the first example. The invariant cohomology of the corresponding variational bicomplex is computed in [8] and a generator of degree 3 appears. Again we show that the closed twoform corresponding to this form does not admit an equivariant moment map by computing the corresponding obstruction in the cohomology of the Lie algebra $\mathfrak{X}\left(S^{1}\right)$ of vector fields on $S^{1}$.

\footnotetext{
*This paper is a contribution to the Special Issue "Élie Cartan and Differential Geometry". The full collection is available at http://www.emis.de/journals/SIGMA/Cartan.html
} 


\section{The integration map}

Let $p: E \rightarrow M$ be a bundle over a compact, oriented $n$-manifold $M$ without boundary, and let $J^{r} E$ be its $r$-jet bundle with projections $p_{r}: J^{r} E \rightarrow M, p_{r, s}: J^{r} E \rightarrow J^{s} E$ for $s<r$. A diffeomorphism $\phi \in \operatorname{Diff} E$ is said to be projectable if there exists $\phi \in \operatorname{Diff} M$ satisfying $\phi \circ p=p \circ \phi$. We denote by $\operatorname{Proj} E$ the space of projectable diffeomorphism of $E$, and we denote by $\operatorname{Proj}^{+} E$ the subgroup of elements such that $\phi \in \operatorname{Diff}^{+} M$, i.e., $\phi$ is orientation preserving. The space of projectable vector fields on $E$ is denoted by $\operatorname{proj} E$, and can be considered as the Lie algebra of ProjE. We denote by $\phi^{(r)}$ (resp. $X^{(r)}$ ) the prolongation of $\phi \in \operatorname{Proj} E$ (resp. $X \in \operatorname{proj} E)$ to $J^{r} E$.

Let $\Gamma(E)$ be the space of global sections of $E$ considered as an infinite dimensional Frechet manifold (e.g. see [6, Section I.4]). For every $s \in \Gamma(E)$ we have $T_{s} \Gamma(E) \cong \Gamma\left(M, s^{*} V(E)\right)$, where $V(E)$ denotes the vertical bundle of $E$. The group Proj $E$ acts naturally on $\Gamma(E)$ in the following way. If $\phi \in \operatorname{Proj} E$, we define $\phi_{\Gamma(E)} \in \operatorname{Diff} \Gamma(E)$ by $\phi_{\Gamma(E)}(s)=\phi \circ s \circ \phi^{-1}$, for all $s \in \Gamma(E)$. In a similar way, a projectable vector field $X \in \operatorname{proj} E$ induces a vector field $X_{\Gamma(E)} \in \mathfrak{X}(\Gamma(E))$.

Let $\mathrm{j}^{r}: M \times \Gamma(E) \rightarrow J^{r} E, \mathrm{j}^{r}(x, s)=j_{x}^{r} s$ be the evaluation map. We define a map

$$
\Im: \Omega^{n+k}\left(J^{r} E\right) \rightarrow \Omega^{k}(\Gamma(E))
$$

by setting

$$
\Im[\alpha]=\int_{M}\left(\mathrm{j}^{r}\right)^{*} \alpha
$$

for $\alpha \in \Omega^{n+k}\left(J^{r} E\right)$. If $\alpha \in \Omega^{k}\left(J^{r} E\right)$ with $k<n$, we set $\Im[\alpha]=0$. The operator $\Im$ satisfies the following properties:

Proposition 1 (cf. [1]). For all $\alpha \in \Omega^{n+k}\left(J^{r} E\right)$ the following formulas hold:

1. $\Im[d \alpha]=d \Im[\alpha]$.

2. $\Im\left[\left(\phi^{(r)}\right)^{*} \alpha\right]=\phi_{\Gamma(E)}^{*} \Im[\alpha]$, for every $\phi \in \operatorname{Proj}^{+} E$.

3. $\Im\left[L_{X^{(r)}} \alpha\right]=L_{X_{\Gamma(E)}} \Im[\alpha]$ for every $X \in \operatorname{proj} E$.

4. $\Im\left[\iota_{X^{(r)}} \alpha\right]=\iota_{X_{\Gamma(E)}} \Im[\alpha]$ for every $X \in \operatorname{proj} E$.

If $\alpha \in \Omega^{n+k}\left(J^{r} E\right), s \in \Gamma(E), X_{1}, \ldots, X_{k} \in T_{s} \Gamma(E) \cong \Gamma\left(M, s^{*} V(E)\right)$, then

$$
\Im[\alpha]_{s}\left(X_{1}, \ldots, X_{k}\right)=\int_{M}\left(j^{r} s\right)^{*}\left(\iota_{X_{k}^{(r)}} \cdots \iota_{X_{1}^{(r)}} \alpha\right) .
$$

There exists a close relationship between the integration map $\Im$ and the variational bicomplex, see [2] for the details.

More generally, if $R \subset J^{r} E$ is an open subset and $\mathcal{R}=\left\{s \in \Gamma(E): j_{x}^{r} s \in R, \forall x \in M\right\}$ is the space of holomonomic sections of $R$, then the integration map defines a map $\Im: \Omega^{n+k}(R) \rightarrow$ $\Omega^{k}(\mathcal{R})$.

\section{Cohomological obstructions to the existence of moment maps}

Recall the obstructions for the existence of a moment map for an invariant closed two-form, e.g., see [7].

Let $\omega$ be a closed two-form on $M$ and $G$ a group acting on $M$ and preserving $\omega$. We have an infinitesimal action $\mathfrak{g} \rightarrow \mathfrak{X}(M), X \mapsto X_{M}$. 
The action is said to be weakly Hamiltonian if for every $X \in \mathfrak{g}$ the form $\iota_{X_{M}} \omega$ is exact, i.e., if there exists a map $\mu: \mathfrak{g} \rightarrow C^{\infty}(M)$ such that for every $X \in \mathfrak{g}$ we have $\iota_{X_{M}} \omega=d(\mu(X))$. The action is said to be Hamiltonian if there exists a $G$-equivariant moment map. At the infinitesimal level, if $\mu: \mathfrak{g} \rightarrow C^{\infty}(M)$ is $G$-equivariant then we have $L_{X} \mu=0$ for every $X \in \mathfrak{g}$ and the converse is true for connected groups.

If the action is weakly Hamiltonian, the obstructions for the action to be Hamiltonian lie in $H^{2}(\mathfrak{g}):$ If $\mu: \mathfrak{g} \rightarrow C^{\infty}(M)$ satisfies $\iota_{X_{M}} \omega=d(\mu(X))$, we define $\tau: \mathfrak{g} \times \mathfrak{g} \rightarrow \mathbb{R}$ by,

$$
\tau(X, Y)=\left(L_{Y} \mu\right)(X)=\mu([X, Y])+L_{Y_{M}}(\mu(X)) .
$$

It can be seen that $\tau$ is closed, that the cohomology class on $H^{2}(\mathfrak{g})$ is independent of the $\mu$ chosen, and that the cohomology class of $\tau$ on $H^{2}(\mathfrak{g})$ vanishes if and only if there exists a moment map $\mu^{\prime}: \mathfrak{g} \rightarrow C^{\infty}(M)$ such that $\iota_{X_{M}} \omega=d\left(\mu^{\prime}(X)\right)$ and $L_{X} \mu^{\prime}=0$ for every $X \in \mathfrak{g}$. In particular, if the cohomology class of $\tau$ is not zero, then the action is not hamiltonian.

\section{Cohomology of smooth vector fields on $S^{1}$}

In our examples we apply the preceding results to the action of the diffeomorphism group of $S^{1}$. Hence the Lie algebra cohomology of $\mathfrak{X}\left(S^{1}\right)$ appears. This cohomology was first computed by Gel'fand and Fuks in [5] and is well known (e.g. see [4]). The continuous cohomology $H\left(\mathfrak{X}\left(S^{1}\right)\right)$ is isomorphic to the tensor product of a polynomial ring with one two-dimensional generator $a$ and the exterior algebra with one three-dimensional generator $b$. The two-dimensional generator $a$ is given by,

$$
a(X, Y)=\int_{S^{1}}\left(\frac{d f}{d t} \frac{d^{2} g}{d t^{2}}-\frac{d g}{d t} \frac{d^{2} f}{d t^{2}}\right) d t
$$

where $X=f(t) \frac{d}{d t}, Y=g(t) \frac{d}{d t}$.

\section{$5 \quad$ Mappings $S^{1} \rightarrow S^{1}$}

In this first example we consider the trivial bundle $E=S^{1} \times S^{1} \rightarrow S^{1}$, whose sections are mappings $u: S^{1} \rightarrow S^{1}$, and the action of $\operatorname{Diff}^{+} S^{1}$ on $E$ by $(\phi,(t, u)) \mapsto(\phi(t), u)$ for $\phi \in \operatorname{Diff}^{+} S^{1}$ and $(t, u) \in S^{1} \times S^{1}$.

The coordinates on $S^{1} \times S^{1}$ are denoted by $(t, u)$ and those on $J^{2} E$ by $(t, u, \dot{u}, \ddot{u})$.

If $X=f(t) \frac{d}{d t} \in \mathfrak{X}\left(S^{1}\right)$, then its prolongation to $J^{2} E$ is given by,

$$
X^{(2)}=f \frac{\partial}{\partial t}-\frac{d f}{d t} \dot{u} \frac{\partial}{\partial \dot{u}}-\left(\frac{d^{2} f}{d t^{2}} \dot{u}+2 \frac{d f}{d t} \ddot{u}\right) \frac{\partial}{\partial \ddot{u}} .
$$

We consider the open subset $\mathcal{A} \subset J^{2} E$ defined by the condition $\dot{u} \neq 0$, which is $\operatorname{Diff}^{+} S^{1}$ invariant, and the form

$$
\sigma=\frac{1}{\dot{u}^{2}} d t \wedge d \dot{u} \wedge d \ddot{u} \in \Omega^{3}(\mathcal{A}) .
$$

It is readily seen that $\sigma$ is closed and that $L_{X^{(2)}} \sigma=0$ for every $X \in \mathfrak{X}\left(S^{1}\right)$. By applying the integration operator $\Im$ we obtain a $\operatorname{Diff}^{+} S^{1}$-invariant closed two-form $\omega=\Im[\sigma] \in \Omega^{2}(\mathfrak{A})$ on the space $\mathfrak{A}$ of holonomic sections of $\mathcal{A}$

$$
\mathfrak{A}=\left\{u: S^{1} \rightarrow S^{1}: \dot{u}(t) \neq 0, \forall t \in S^{1}\right\} .
$$


In our case, if $s: S^{1} \rightarrow S^{1} \times S^{1}$ is the section corresponding to $u: S^{1} \rightarrow S^{1}$, then $T_{s} \Gamma(\mathcal{A}) \cong$ $\Gamma\left(S^{1}, s^{*} V(E)\right) \cong \Gamma\left(S^{1}, T S^{1}\right)$.

In local coordinates, if $H=h(t) \frac{\partial}{\partial u}$, then its prolongation to $J^{2} E$ is given by,

$$
H^{(2)}=h \frac{\partial}{\partial u}+\frac{d h}{d t} \frac{\partial}{\partial \dot{u}}+\frac{d^{2} h}{d t^{2}} \frac{\partial}{\partial \ddot{u}} .
$$

Using this expression and formula (2) we obtain the explicit expression of $\omega$ :

Proposition 2. If $H, K \in T_{s} \Gamma(\mathcal{A})$ are given by $H=h(t) \frac{\partial}{\partial u}, K=k(t) \frac{\partial}{\partial u}$, and $u: S^{1} \rightarrow S^{1}$ then

$$
\omega_{u}(H, K)=\int_{S^{1}}\left(\frac{d u}{d t}\right)^{-2}\left(\frac{d^{2} h}{d t^{2}} \frac{d k}{d t}-\frac{d h}{d t} \frac{d^{2} k}{d t^{2}}\right) d t .
$$

Moreover, we have $\sigma=d \alpha$, where $\alpha=\dot{u}^{-1} d t \wedge d \ddot{u}$, and then,

$$
\iota_{X^{(2)}} \sigma=\iota_{X^{(2)}} d \alpha=L_{X^{(2)}} \alpha-d\left(\iota_{X^{(2)}} \alpha\right),
$$

for all $X \in \mathfrak{X}\left(S^{1}\right)$. By using (2) we also obtain,

$$
L_{X^{(2)}} \alpha=-\frac{d^{2} f}{d t^{2}} \frac{1}{\dot{u}} d t \wedge d \dot{u}=d\left(-\frac{d f}{d t} \frac{d \dot{u}}{\dot{u}}\right)
$$

and hence,

$$
\iota_{X^{(2)}} \sigma=d\left(-\frac{d f}{d t} \frac{d \dot{u}}{\dot{u}}-\iota_{X^{(2)}} \alpha\right)=d(\rho(X)),
$$

where

$$
\rho(X)=-\frac{d f}{d t} \frac{d \dot{u}}{\dot{u}}-\iota_{X^{(2)}} \alpha .
$$

Accordingly, the action of $\operatorname{Diff}^{+} S^{1}$ on $(\mathfrak{A}, \omega)$ is weakly Hamiltonian with moment map $\mu(X)=$ $\Im[\rho(X)], \forall X \in \mathfrak{X}\left(S^{1}\right)$, as we have

$$
\iota_{X_{\mathfrak{A}}} \omega=\iota_{X_{\mathfrak{A}}} \Im[\sigma]=\Im\left[\iota_{X^{(2)}} \sigma\right]=\Im[d(\rho(X))]=d(\Im[\rho(X)])=d(\mu(X)) .
$$

The explicit expression of $\mu$ is the following:

Proposition 3. If $X=f(t) \frac{d}{d t}$ and $u: S^{1} \rightarrow S^{1}$, then

$$
\mu(X)_{u}=-\int_{S^{1}}\left(\frac{d u}{d t}\right)^{-1}\left(f \frac{d^{3} u}{d t^{3}}+3 \frac{d f}{d t} \frac{d^{2} u}{d t^{2}}+\frac{d^{2} f}{d t^{2}} \frac{d u}{d t}\right) d t
$$

However, the action is not Hamiltonian.

Proposition 4. If $X=f(t) \frac{d}{d t}, Y=g(t) \frac{d}{d t}$, then

$$
\tau(X, Y)=-\int_{S^{1}}\left(\frac{d^{2} f}{d t^{2}} \frac{d g}{d t}-\frac{d f}{d t} \frac{d^{2} g}{d t^{2}}\right) d t .
$$

Proof. From the definition of $\tau$ we have

$$
\begin{aligned}
\tau(X, Y) & =L_{Y_{\mathfrak{A}}} \mu(X)+\mu([X, Y])=\Im\left[L_{Y^{(2)}} \rho(X)\right]+\Im[\rho([X, Y])] \\
& \left.=\Im\left[L_{Y^{(2)}} \rho X\right)+\rho([X, Y])\right] .
\end{aligned}
$$


From the definition of $\rho$ we have

$$
\begin{aligned}
L_{Y^{(2)}} \rho(X)+\rho([X, Y])= & L_{Y^{(2)}}\left(-\frac{d x}{d t} \frac{d \dot{u}}{\dot{u}}\right)-L_{Y^{(2)}}\left(\iota_{X^{(2)}} \alpha\right) \\
& -\frac{d}{d t}\left(f \frac{d g}{d t}-f \frac{d g}{d t}\right) \frac{d \dot{u}}{\dot{u}}-\iota_{\left[X^{(2)}, Y^{(2)}\right]} \alpha .
\end{aligned}
$$

By using (2) we obtain

$$
\begin{aligned}
& L_{Y^{(2)}}\left(\iota_{X^{(2)}} \alpha\right)+\iota_{\left[X^{(2)}, Y^{(2)}\right]} \alpha=\iota_{X^{(2)}}\left(L_{Y^{(2)}} \alpha\right)=-f \frac{d^{2} g}{d t^{2}} \frac{d \dot{u}}{\dot{u}}-\frac{d f}{d t} \frac{d^{2} g}{d t^{2}} d t, \\
& L_{Y^{(2)}}\left(-\frac{d f}{d t} \frac{d \dot{u}}{\dot{u}}\right)=-g \frac{d^{2} f}{d t^{2}} \frac{d \dot{u}}{\dot{u}}+\frac{d f}{d t} \frac{d^{2} g}{d t^{2}} d t \\
& \frac{d}{d t}\left(f \frac{d g}{d t}-g \frac{d f}{d t}\right) \frac{d \dot{u}}{\dot{u}}=\left(f \frac{d^{2} g}{d t^{2}}-g \frac{d^{2} f}{d t^{2}}\right) \frac{d \dot{u}}{\dot{u}},
\end{aligned}
$$

and hence,

$$
L_{Y^{(2)}} \rho(X)+\rho([X, Y])=2 \frac{d f}{d t} \frac{d^{2} g}{d t^{2}} d t .
$$

As

$$
2 \frac{d f}{d t} \frac{d^{2} g}{d t^{2}} d t=-\left(\frac{d^{2} f}{d t^{2}} \frac{d g}{d t}-\frac{d f}{d t} \frac{d^{2} g}{d t^{2}}\right) d t+d\left(\frac{d f}{d t} \frac{d g}{d t}\right),
$$

we finally obtain,

$$
\tau(X, Y)=\int_{S^{1}} 2 \frac{d f}{d t} \frac{d^{2} g}{d t^{2}} d t=-\int_{S^{1}}\left(\frac{d^{2} f}{d t^{2}} \frac{d g}{d t}-\frac{d f}{d t} \frac{d^{2} g}{d t^{2}}\right) d t
$$

Accordingly to Section 4 the expression obtained in Proposition 5 determines a non-trivial class on the Lie algebra cohomology of $\mathfrak{X}\left(S^{1}\right)$, and hence $\langle\tau\rangle \neq 0$ in $H^{2}\left(\mathfrak{X}\left(S^{1}\right)\right)$. Hence we obtain the following

Corollary 1. The action of $\operatorname{Diff}^{+} S^{1}$ on $(\mathfrak{A}, \omega)$ is not Hamitonian, i.e., $\omega$ does not admit $a$ Diff $^{+} S^{1}$-equivariant moment map.

\section{Regular plane curves}

Let $E=S^{1} \times \mathbb{R}^{2} \rightarrow S^{1}$ be the trivial bundle. Global sections of $E$ are none other than mappings $u: S^{1} \rightarrow \mathbb{R}^{2}, u(t)=(x(t), y(t))$. Coordinates on $E$ are denoted by $(t, x, y)$ and by $(t, x, y, \dot{x}, \dot{y}, \ddot{x}, \ddot{y})$ the coordinates on $J^{2} E$.

We consider the open set $\mathcal{R} \subset J^{2} E$ defined by the condition $\dot{x}^{2}+\dot{y}^{2} \neq 0$, and corresponding to the 2 -jets of regular curves. The holonomic sections of $\mathcal{R}$ constitute the space $\mathfrak{R e g} \subset \Gamma(E)$ of closed regular plane curves,

$$
\mathfrak{R e g}=\left\{u: S^{1} \rightarrow \mathbb{R}^{2}:|\dot{u}(t)|^{2} \neq 0, \forall t \in S^{1}\right\} .
$$

The group Diff $S^{1}$ acts on $E$ by $(t, u) \mapsto(\phi(t), u)$, for every $\phi \in \operatorname{Diff} S^{1}$. This action induces an action on $J^{2} E$ and clearly $\mathcal{R}$ is invariant under this action. If $X=f \frac{d}{d t} \in \mathfrak{X}\left(S^{1}\right)$, then its prolongation to $J^{2} E$ is given by

$$
X^{(2)}=f \frac{\partial}{\partial t}-\frac{d f}{d t} \dot{x} \frac{\partial}{\partial \dot{x}}-\frac{d f}{d t} \dot{y} \frac{\partial}{\partial \dot{y}}-\left(\frac{d^{2} f}{d t^{2}} \dot{x}+2 \frac{d f}{d t} \ddot{x}\right) \frac{\partial}{\partial \ddot{x}}-\left(\frac{d^{2} f}{d t^{2}} \dot{y}+2 \frac{d f}{d t} \ddot{y}\right) \frac{\partial}{\partial \ddot{y}} .
$$


Let us consider the 3 -form on $\mathcal{R}$ given by

$$
\sigma=v^{-2} d t \wedge d v \wedge d \dot{v}
$$

where $v=\sqrt{\dot{x}^{2}+\dot{y}^{2}}, \dot{v}=v^{-1}(\dot{x} \ddot{x}+\dot{y} \ddot{y})$. This form appears in [8] as a generator of the cohomology for the invariant cohomology of the variational bicomplex for regular plane curves. It is easily checked directly that $L_{X^{(2)}} \sigma=0$ for every $X \in \mathfrak{X}\left(S^{1}\right)$ and that $d \sigma=0$.

Let $\omega=\Im[\sigma] \in \Omega^{2}(\mathfrak{R})$, where $\Im: \Omega^{3}(\mathcal{R}) \rightarrow \Omega^{2}(\mathfrak{R e g})$ is the integration map. By the properties of $\Im$, we know that $\omega$ is a closed and Diff ${ }^{+} S^{1}$-invariant two-form on $\mathfrak{R e g}$.

We apply the results of Section 3 to the - infinite-dimensional - case of the $\operatorname{Diff}^{+} S^{1}$-action on $(\mathfrak{R e g}, \omega)$.

Let $\alpha \in \Omega^{2}(\mathcal{R})$ be the form given by, $\alpha=v^{-1} d t \wedge d \dot{v}$. Clearly, we have $d \alpha=\sigma$, and hence $\iota_{X^{(2)}} \sigma=\iota_{X^{(2)}} d \alpha=L_{X^{(2)}} \alpha-d\left(\iota_{X^{(2)}} \alpha\right)$, for every $X \in \mathfrak{X}\left(S^{1}\right)$.

As a direct computation shows, we have

$$
\begin{aligned}
L_{X^{(2)}} v & =-\frac{d f}{d t} v, \\
L_{X^{(2)}} \dot{v} & =-\frac{d^{2} f}{d t^{2}} v-2 \frac{d f}{d t} \dot{v},
\end{aligned}
$$

and hence

$$
L_{X^{(2)}} \alpha=-\frac{d^{2} f}{d t^{2}} d t \wedge \frac{d v}{v}=-d\left(\frac{d f}{d t}\right) \wedge \frac{d v}{v}=d\left(-\frac{d f}{d t} \frac{d v}{v}\right) .
$$

Hence we obtain

$$
\iota_{X^{(2)}} \sigma=d\left(-\frac{d f}{d t} \frac{d v}{v}\right)-d\left(\iota_{X^{(2)}} \alpha\right)=d\left(-\frac{d f}{d t} \frac{d v}{v}-\iota_{X^{(2)}} \alpha\right) .
$$

If we set

$$
\rho(X)=-\frac{d f}{d t} \frac{d v}{v}-\iota_{X^{(2)}} \alpha,
$$

then the action is weakly Hamiltonian, with moment map

$$
\mu(X)=\Im[\rho(X)], \quad \forall X \in \mathfrak{X}\left(S^{1}\right) .
$$

However, the action is not Hamiltonian.

Proposition 5. If $X=f(t) \frac{d}{d t}, Y=g(t) \frac{d}{d t}$, then

$$
\tau(X, Y)=-\int_{S^{1}}\left(\frac{d^{2} f}{d t^{2}} \frac{d g}{d t}-\frac{d f}{d t} \frac{d^{2} g}{d t^{2}}\right) d t .
$$

Proof. We have

$$
\begin{aligned}
\tau(X, Y) & =L_{Y_{\mathfrak{R e g}}} \mu(X)+\mu([X, Y])=\Im\left[L_{Y^{(2)}} \rho(X)\right]+\Im[\rho([X, Y])] \\
& =\Im\left[L_{Y^{(2)}} \rho(X)+\rho([X, Y])\right]
\end{aligned}
$$

and

$$
\begin{aligned}
L_{Y^{(2)}} \rho(X)+\rho([X, Y])= & L_{Y^{(2)}}\left(-\frac{d f}{d t} \frac{d v}{v}\right)-L_{Y^{(2)}}\left(\iota_{X^{(2)}} \alpha\right) \\
& -\frac{d}{d t}\left(f \frac{d g}{d t}-g \frac{d f}{d t}\right) \frac{d v}{v}-\iota_{\left[X^{(2)}, Y^{(2)}\right]} \alpha .
\end{aligned}
$$


By using (3), (4), and (5) we obtain

$$
\begin{aligned}
& L_{Y^{(2)}}\left(\iota_{X^{(2)}} \alpha\right)+\iota_{\left[X^{(2)}, Y^{(2)}\right]} \alpha=\iota_{X^{(2)}}\left(L_{Y^{(2)}} \alpha\right)=\frac{d^{2} g}{d t^{2}}\left(-f \frac{d v}{v}-\frac{d f}{d t} d t\right), \\
& L_{Y^{(2)}}\left(-\frac{d f}{d t} \frac{d v}{v}\right)=-\frac{d^{2} f}{d t^{2}} g \frac{d v}{v}+\frac{d f}{d t} \frac{d^{2} g}{d t^{2}} d t, \\
& \frac{d}{d t}\left(f \frac{d g}{d t}-g \frac{d f}{d t}\right) \frac{d v}{v}=\left(f \frac{d^{2} g}{d t^{2}}-g \frac{d^{2} f}{d t^{2}}\right) \frac{d v}{v},
\end{aligned}
$$

and hence,

$$
L_{Y^{(2)}} \rho(X)+\rho([X, Y])=2 \frac{d f}{d t} \frac{d^{2} g}{d t^{2}} d t .
$$

As

$$
2 \frac{d f}{d t} \frac{d^{2} g}{d t^{2}} d t=-\left(\frac{d^{2} f}{d t^{2}} \frac{d g}{d t}-\frac{d f}{d t} \frac{d^{2} g}{d t^{2}}\right) d t+d\left(\frac{d f}{d t} \frac{d g}{d t}\right)
$$

we finally obtain

$$
\tau(X, Y)=\int_{S^{1}} 2 \frac{d f}{d t} \frac{d^{2} g}{d t^{2}} d t=-\int_{S^{1}}\left(\frac{d^{2} f}{d t^{2}} \frac{d g}{d t}-\frac{d f}{d t} \frac{d^{2} g}{d t^{2}}\right) d t
$$

As we obtain the same result as that in the preceding example, we also obtain the following

Corollary 2. The action of $\operatorname{Diff}^{+} S^{1}$ on $(\mathfrak{R e g}, \omega)$ is not Hamitonian, i.e., $\omega$ does not admit a Diff ${ }^{+} S^{1}$-equivariant moment map.

\section{Acknowledgements}

Part of this work started during the stay of the first author at Utah State University under the advice of Professor Ian Anderson. The computations were first obtained by using MAPLE package "Vessiot". Supported by Ministerio de Ciencia e Innovación of Spain under grant \# MTM2008-01386.

\section{References}

[1] Ferreiro Pérez R., Equivariant characteristic forms in the bundle of connections, J. Geom. Phys. 54 (2005), 197-212, math-ph/0307022.

[2] Ferreiro Pérez R., Local cohomology and the variational bicomplex, Int. J. Geom. Methods Mod. Phys. 5 (2008), 587-604.

[3] Ferreiro Pérez R., Muñoz Masqué J., Pontryagin forms on (4k-2)-manifolds and symplectic structures on the spaces of Riemannian metrics, math.DG/0507076.

[4] Fuks D.B., Cohomology of infinite-dimensional Lie algebras, Contemporary Soviet Mathematics, Consultants Bureau, New York, 1986.

[5] Gel'fand I.M., Fuks D.B., Cohomologies of the Lie algebra of vector fields on the circle, Funkcional. Anal. i Prilozen. 2 (1968), no. 4, 92-93.

[6] Hamilton R., The inverse function theorem of Nash and Moser, Bull. Amer. Math. Soc. (N.S.) 7 (1982), 65-222.

[7] McDuff D., Salamon D., Introduction to symplectic topology, Oxford Mathematical Monographs, Oxford Science Publications, The Clarendon Press, Oxford University Press, New York, 1995, 1995.

[8] Pohjanpelto J., Anderson I.M., Infinite-dimensional Lie algebra cohomology and the cohomology of invariant Euler-Lagrange complexes: a preliminary report, in Differential Geometry and Applications (Brno, 1995), Masaryk Univ., Brno, 1996, 427-448. 